\title{
Dielectric constant and chlorophyll content measurements for basal stem rot (BSR) disease detection
}

\begin{abstract}
Basal stem rot (BSR) is a common plant disease that is largely responsible for high economic losses in oil palm production. Several novel techniques have recently been develop and reported in the literature for detecting BSR disease in oil palm plantations. However, studies on the application of electrical properties in detecting BSR disease in oil palm does not exist. Therefore, this paper aims to contribute to the existing knowledge by investigating the potential of dielectric constant (DC) and chlorophyll properties in detecting BSR disease in oil palms. The study involved the collection of different leaf samples namely; healthy, mild, moderate, and severely-infected. Impedance analyzer operating at a frequency range of 100 kHz-30 MHz with 300 spectral intervals and SPAD 502 were used to measure the DC and chlorophyll of the samples collected, respectively. ANOVA, Duncan's multiple range test (DMRT) and principal component analysis (PCA) were used for statistical analysis. The results of this study showed a significant relationship between DC and different severity levels of BSR disease ( $\mathrm{p}<;$ 0.0001). Specifically, BSR disease severity levels of all samples collected were clearly discriminated based on DC. Conversely, the chlorophyll content could not classify the different levels of BSR disease into distinct separate groups but two groups (healthy and BSR-infected). As such, the results demonstrated that DC and chlorophyll content at certain extend could be used as a sensing parameter for Ganoderma disease detection.
\end{abstract}

Keyword: Basal stem rot; Chlorophyll; Dielectric constant; Principal component analysis 